\title{
A dynamic highway flow control simulation model for solving the congestion problem
}

\author{
C. L. Yang ${ }^{1} \&$ W. Wen ${ }^{2}$ \\ ${ }^{I}$ Department of Information Management, Tatung University, \\ Taiwan, R.O.C. \\ ${ }^{2}$ Department of Information Management, \\ LungHwa University of Science and Technology, Taiwan, R.O.C.
}

\begin{abstract}
In this paper, we have developed a simulation model coded in Arena to help design a dynamically automatic traffic flow control simulation model without a time limit barrier. The model simulates the section of the Chung-Sam highway between Taipei and TaoYuan in which the distance is 31.9 kilometers. We adopt dynamic speed limits and practical measures to simulate the arrival number of cars and the leaving number of cars in a time interval. First, we set each lane with the speed limit of 100 or $125 \mathrm{~km} / \mathrm{hr}$ and a stop-and-go traffic light sign at the entrance ramp with $0,5,10$, and 15 seconds. Next, by controlling the departure speed of the junction, we find that it causes a better result for enlarging the maximum flow. The simulation model with the dynamic speed limit is able to dynamically guide traffic in rush hours. Finally, the dynamically automatic traffic flow control models without a time limit barrier and with a time limit barrier at $0,5,10$, and 15 seconds are implemented and analyzed. We find that the dynamically automatic traffic flow control model without a time limit barrier is better than that of the model with a time limit barrier.
\end{abstract}

Keywords: simulation model, highway traffic, traffic congestion.

\section{Introduction}

Highway traffic congestion has been causing many critical problems and challenges in many modern cities, which have highways to pass through them. In particular, at rush hours, drivers, who need to go to offices daily via a highway, do not like to queue for long periods at entrance ramps or on highways. To the 
commuter or traveller, congestion means lost time, missed opportunities, and frustration. To the employer, congestion means lost worker productivity, trade opportunities, delivery delays, and increased costs. A growing body of evidence proves that traffic congestion problems cannot be solved simply by expanding road infrastructure. In particular, to construct a new highway around a big city is almost not possible because of high cost of land and difficulty to get it. Therefore, many countries are working to manage their existing transportation systems to improve mobility, safety, and traffic flows and to reduce demand for vehicle use. From recent analytical statistics [14], it is estimated that roughly half of the congestion is what is known as recurring congestion - caused by recurring demands that exist virtually every day, where road use exceeds existing capacity. The other half is due to non-recurring congestion caused by temporary disruptions. Researchers have done many researches to increase capacity and remove bottlenecks.

Hall and Chin [6] developed and evaluated strategies for grouping vehicles into platoon at highway entrances. Platoon means that vehicles travel on highways in closely distance, 1 meter, within a group but the distance between platoons is large for safe reasons. In their result, large size of platoons and long distances can get maximum throughput. In order to get the objective, sorting vehicles by destination at the entrance of highways is one of good methods. Schadschneider [11] used cellular automata (CA) models to simulate interdisciplinary problems for the description of highway traffic. CA models have the ability to reproduce the empirically observed structure of traffic flow on a microscopic level. Other research papers relating to simulation models to solve highway traffic problems are found in [7,8,12,15]. Alvarez et al. [1] presented a multi-destination traffic flow control in which vehicles have different destinations and types in automated highway system for increasing capacity and safety. Their paper focuses on control strategies at the macroscopic level of traffic where the quantities are the aggregate vehicular density and the traffic flow measured in sections of highway. Golob and Recker [4] developed a method to decide how crash characteristics are related to traffic flow conditions. A case study was adopted and identified twenty-one traffic flow regimes for three different ambient conditions: dry roads during daylight, dry roads at night, and wet conditions. The paper concludes that congestion deeply affects highway safety. Schaefer et al. [12] developed a simulation model for evaluating freeway lane control signing. The simulation results show that lane control has little influence on congestion. However, the region between heavy and medium traffic flow is sensitive to lane control. Qin et al. [10] divided crashes into four types: single-vehicle, multi-vehicle same direction, and multi-vehicle opposite direction. Their results show that for single-vehicle crashes, the marginal crash rate is high at low traffic volumes and low at high traffic volumes. However, for the multi-vehicle same direction, the relationship is reverse. The crashes of the multi-vehicle opposite direction increase as a linear with the traffic volumes. Chen and Yang [2,3] created an algorithm to find the minimum total time path to simulate the operations of traffic-light control in a city. Stoilova and Stoilov [13] also built a simulation model to measure the best of traffic lights to achieve low 
noise levels with optimal traffic management and environmental pollution. Grau and Barcelo [5] and Messmer and Papageorgiou [9] discussed the minimum of queue lengths in different intersections. Our research focuses on increasing speed limit on highways or reducing stop-and-go traffic light durations to improve highway congestion problem, which will be discussed below.

\section{The framework for a dynamic highway traffic control simulation model}

The highway traffic simulation model is designed to cover a length of $31.9 \mathrm{~km}$ in the Chungsan highway, Taipei, Taiwan, R.O.C. starting from junction Yuansan, Taipei, to junction Taoyuan as our target section because it is always busy in the rush hours from 7:30 am to 8:30 am. The model for simplicity only covers 3 junctions. In the framework for the simulation model shown as Figure 1, assumes that the highway has 3 junctions consisting of a starting junction (junction 1), an exit junction (junction 2), and an end junction (junction 3). In the model, we assume that there is a stop-and-go traffic light sign at the starting junction (junction1).

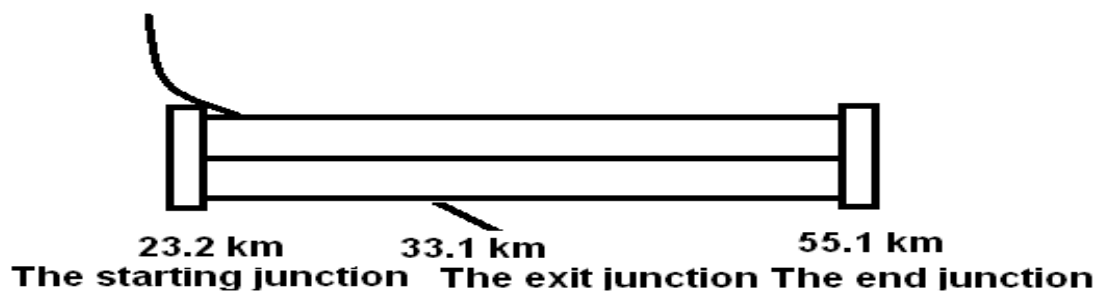

Figure 1: A framework for dynamic highway traffic control simulation model.

\subsection{Definitions and notations of the simulation model}

To illustrate how the simulation model works, we have designed a highway traffic simulation model by using Arena. Before giving example, let us introduce the definition of notations as follows:

Variables: (The value will not be carried when a car moves)

Lane1No: The number of cars in lane 1.

Lane2No: The number of cars in lane 2.

Lane3No: The number of cars in lane 3.

Drive1Time: The checking time in lane 1 for deciding whether to change lane.

Drive2Time: The checking time in lane 2 for deciding whether to change lane.

Drive3Time: The checking time in lane 3 for deciding whether to change lane.

Junction2Dis: The distance from the starting junction to junction 2.

SystemDis: The distance from the starting junction to junction 3 .

Lane1ExitNo: Total number of cars in lane 1 to exit the end junction. 
Lane2ExitNo: Total number of cars in lane 2 to exit the end junction.

Lane3ExitNo: Total number of cars in lane 3 to exit the end junction.

Junction2ExitNo: Total number of cars from junction 2 to exit.

TotalCarsNo: The total number of cars exiting from lane 1, lane 2, 3, and junction 2. (Lane1ExitNo +Lane2ExitNo+ Lane3ExitNo+ Junction2ExitNo).

CarLength: The length of a car, which is 4.7 meters.

SafeLength: The safe distance between cars. (Basically, each car speed increases $10 \mathrm{~km} / \mathrm{hr}$ and its safe length needs to add a length of a car.)

TotalRampNo: The total car numbers from an entrance ramp into lane 1 .

Attributes: (The values are carried by a car generated by modules, Create lane1 cars arrival, Create lane 2 cars arrival, Create lane 3 cars arrival, and Create entrance ramp cars arrival, when the car moves.)

DriveDistance: The travelled distance of a car.

Speed1: The average speed of cars in lane1.

Speed2: The average speed of cars in lane2.

Speed3: The average speed of cars in lane3.

\subsection{Description of the simulation model}

Figure 2 shows the flow chart of the highway traffic flow control simulation model. To illustrate the simulation model in detail, we explain the flow chart step by step. However, due to the similarity, we only describe the process of lane 1 and omit the process of lanes 2 and 3. In the model, a stop-and-go traffic light signal for allowing cars to enter into the lane of the highway from an entranceramp can be controlled by a module named Create light control. Besides, cars in each lane named entities are created and based on cars' arrival time set in modules, Create lane1 cars arrival, Create lane 2 cars arrival, and Create lane 3 cars arrival.

Basically, the module, Create entrance ramp cars arrival, needs to seize a resource, switch, for a car to pass a stop line at the starting junction for driving into lane 1 of the highway. In other words, a car (i.e., an entity) needs to pass the stop line at the entrance ramp in the junction 1 if only if the car seizes the resource. Without seizing the resource, switch, the car will be put in a queue and can do nothing but waiting. Therefore a car and a light control compete with each other in order to get a resource. However, in order to let the Create light control module always get a resource, switch, we set the highest priority for the Create light control module and cars with a lower priority. Once the Create light control module gets the switch, it will delay $0,5,10$, or 15 seconds for red light to stop any car entering into the highway. Then, the Create light control module releases the switch and delays another 15 seconds for green light. When the Create light control module releases the switch, Create entrance ramp cars arrival can seize the switch and delays 1.2 seconds for letting cars enter the highway. The interarrival time for the Create entrance ramp cars arrival module is 1.72 seconds. Finally, if any car drives from the entrance ramp into the lane 1 of the highway, the number of cars, Lane1No, in lane 1 will be added 
by 1 and the Release switch for next car module will release the switch for waiting the next car to seize it. The process is continuing until the Create light control module turns red because it has the highest priority. During the red light period, no any car is allowed to pass the stop line because it is unable to get the switch to have rights entering into the highway. Before merging to the Create lane1 cars arrival module, the module, Assign entrance ramp cars number increasing, acuminates how many cars have entered into lane 1 by using the equation, TotalRampNo $=$ TotalRampNo +1 .

The module, Create lane1 cars arrival, generates cars (i.e., entities) for lane 1. The interarrival time for the module, Create lane1 cars arrival, is 2.1 seconds. The Assign initial lane1 driven distance module sets the initial value of the attribute, DriverDistance, which is 0 . Then, the Station lane 1 module is an accepting point when other modules call it. The Assign lane 1 cars number increasing module computes the number of Lane1No by using the equation, Lane1No=Lane1No +1 . Note that the Assign lane 1 speed module gives a dynamic speed based on the equations, SafeLength=SystemDis/ $($ CarLength $*$ Lane1No) -1 and Speed1 $=$ SafeLength $* 10 * 1000 / 3600$. It implies that the more cars in each lane, the slower speed in each lane. If the Decide below maximum speed1 module is true, then let Speed1 $=34.7 \mathrm{~m} / \mathrm{sec}$ (i.e., 125 $\mathrm{km} / \mathrm{hr}$ ) and if the Decide below maximum speed1 module is false, then the Assign lane1 maximum speed module sets Speed1 $=33.7 \mathrm{~m} / \mathrm{sec}$. The Delay lane1 drive time module represents that the system delays 5 seconds (i.e., Drive1 Time=5) to let the next module, Assign lane1 driven distance, count the distance, which the car travelled. The module, Assign lane1 driven distance, computes the distance that the car travelled by using the equation, DriveDistance $=$ DriveDistance + Drive1Time $*$ Speed1. The Decide to stay lane1 or lane 2 module sets that $20 \%$ of total cars in lane 1 will randomly change lane to lane 2 and $80 \%$ of total cars in lane 1 will keep the current lane, lane 1. If the module, Decide to stay lane1 or lane2, is true, then the Assign lane1_1 cars number decreasing module lets Lane1No be reduced by 1 as the following equation, Lane1No=Lane1No - 1 . Then, the car is assigned to lane2, which means that it goes to the Station lane2 module. However, if the module, Decide to stay lane1 or lane2, is false, then the Decide arriving junction2 module needs to check whether the car arrives at the exit junction, junction 2 , based on the condition, DriveDistance $\geq$ Junction2Dis.

When the car has not reached junction 2, it returns to the Assign lane 1 speed module. When the car reaches junction 2, the Decide off junction2 module decides whether the car needs to exit junction 2 or not. If the module, Decide off junction2, is true, which is $20 \%$, then the module, Assign lane1_2 cars number decreasing, is reduced by 1 according to the equation, lane1No=lane1No - 1 , and the Assign junction2 exit cars number module increases Junction2ExitNo by 1 (i.e., Junction2ExitNo= Junction2ExitNo+1). The module, Assign total_1 cars exit number, computes the total number of cars that exit from lane 1, lane 2, lane 3, and junction2 (i.e., TotalCarsNo=Lane1ExitNo+Lane2ExitNo+Lane3ExitNo +Junction2Exit No). Finally, the car leaves the system by using the Dispose lane1_sec1 module. 
If the Decide off junction2 module is false (i.e., $80 \%$ of the total number in lane 1), then the Station lane1_1 module lets the car return to the module, Station lane1.

The Delay lane1_sec2 drive time module delays 5 seconds for computing the current travelled distance. Therefore, the Assign lane1_sec2 driven distance module counts the current travelled distance using DriveDistance $=$ DriveDistance + Drive1Time*Speed1. The Decide to stay lane1_sec2 or lane 2 module sets $20 \%$ of total cars in lane 1 to lane 2 and $80 \%$ of total cars in lane 1 keep in lane 1. If Decide to stay lane1_sec2 or lane2 is true, then the Assign lane1_3 cars number decreasing module decreases by 1 as Lane1 No=Lane1No -1 . Next, the car returns to the Station lane 2 module. But when the Decide off junction2 module determines that the car needs to exit junction 2, then the Assign lane1_4 cars number decreasing module reduces Lane1No by 1 (i.e., Lane1No =Lane1No-1). Then, the Assign lane1 exit cars number module increases by 1 (i.e., Lane1ExitNo= Lane1ExitNo+1). Finally, the car is discarded for representing to exit the system based on the Dispose lane1_sec2 module. If Decide off junction2 is false, then the car returns to the Station lane1 module.

The Assign lane 1_sec2 speed module similarly gives a dynamic speed as mentioned earlier according to the equations, SafeLength=SystemDis/ (CarLength*Lane1No)-1 and Speed1 $=$ SafeLength*10*1000/3600. If the Decide below maximum speed1_1 module is true, then let $\mathbf{S p e e d 1}=34.7 \mathrm{~m} / \mathrm{sec}$ (i.e., $125 \mathrm{~km} / \mathrm{hr}$ ) and if the Decide below maximum speed1_1 module is false, then the Assign lane1_sec2 maximum speed module sets Speed1=33.7 m/sec. The Delay lane1_sec2 drive time module represents that the system delays 5 seconds, which means Drive1Time $=5$, to let the next module, Assign lane1_sec2 driven distance, count the distance that the car travelled. The module, Assign lane1_sec2 driven distance, computes the distance that the car travelled by using the equation, DriveDistance $=$ DriveDistance + Drive1Time *Speed1. The Decide to stay lane1_sec2 or lane2 module sets that $20 \%$ of total cars in lane 1 will randomly change lane to lane 2 and $80 \%$ of total cars in lane 1 will keep the current lane, lane 1. If the module, Decide to stay lane1_sec2 or lane2, is true, then the Assign lane1_3 cars number decreasing module lets Lane1No be reduced by 1 using the following equation, Lane1No=Lane1No 1. Then, the car is assigned to lane2, which means that it goes to the Station lane2 module. However, if the module, Decide to stay lane1_sec2 or lane2, is false, then the Decide leaving the system module needs to check whether the car arrives at the end junction, junction 3, based on the condition, DriveDistance $\geq$ SystemDis. When the car has not reached junction 3, it returns to the point before the Assign lane 1_sec2 speed module. When the car reaches junction 3, then the module, Assign lane1 4 cars number decreasing, is reduced by 1 according to the equation, Lane1No=Lane1No -1 , and the Assign lane1 cars exit number module increases Lane1ExitNo by 1 (i.e., Lane1ExitNo= Lane1ExitNo+1). The module, Assign total_2 cars exit number, computes the total number of cars that exit from lane 1, lane 2, lane 3, and junction2 (i.e., TotalCarsNo=Lane1ExitNo+Lane2ExitNo+Lane3ExitNo+ 
Junction2ExitNo). Finally, the car leaves the system by using the Dispose lane1_sec2 module.

The Delay lane1_sec2 drive time module delays 5 seconds for computing the current travelled distance. Therefore, the Assign lane1_sec2 driven distance module counts the current travelled distance using DriveDistance $=$ DriveDistance + Drive1Time*Speed1. The Decide to stay lane1_sec2 or lane 2 module sets $20 \%$ of total cars in lane 1 to lane 2 and $80 \%$ of total cars in lane 1 keep in lane 1. If Decide to stay lane1_sec2 or lane2 is true, then the Assign lane1_3 cars number decreasing module decreases by 1 using the equation, Lane1No=Lane1No -1 . Next, the car returns to the Station lane2 module. But when the Decide off junction2 module decides that the car needs to exit from junction 2, then the Assign lane1_4 cars number decreasing module reduces Lane1No by 1 (i.e., Lane1No = Lane1No-1). Then, the Assign lane1 exit cars number module increases by 1 (i.e., Lane1ExitNo= Lane1ExitNo+1). Finally, the car is discarded for representing to exit the system in according with the Dispose lane1_sec2 module. If the Decide off junction2 module is false, then the car returns to the Delay lane1_sec2 drive time module.

\section{Simulation analysis and results}

Using Arena, we have built the dynamic highway flow control simulation model. During the simulation analysis, initially a run time of 3600 seconds is set. In the model, a stop-and-go traffic light sign is installed for controlling the car number to enter into the highway. According to a practical measure, we assume the green light duration with 15 seconds. However, for the red light duration, it starts from 0 second and then is increased by 5 seconds each time (i.e., 0, 5, 10, and 15 seconds). Also, the interarrival time of each car in the lanes 1, 2, and 3 is 2.1 seconds. For understanding performance, we fix the speed limit in each lane to 100 and $125 \mathrm{~km} / \mathrm{hr}$ and compare the current car number and average speed in each lane. The current car number presents the total car number in lanes 1, 2, or 3 . From Table 1 with a speed limit of $100 \mathrm{~km} / \mathrm{hr}$, the current car number in lanes 1,2 , or 3 is smaller than that of lanes 1,2 , or 3 with a speed limit of $125 \mathrm{~km} / \mathrm{hr}$. However, there is an exception in lane 1 . The current car number, 1341, in lane 1 with a speed limit of $100 \mathrm{~km} / \mathrm{hr}$, is a little bite bigger than that, 1337, of lane 1 with a speed limit of $125 \mathrm{~km} / \mathrm{hr}$.

With respect to the average speed, the average speed in each lane with 100 $\mathrm{km} / \mathrm{hr}$ is smaller than that of each lane with $125 \mathrm{~km} / \mathrm{hr}$. In other words, if we increase the speed limit of each lane, then the average speed in each lane will also increase. Meanwhile, the current car number in each lane will decrease. Another very important message is that the total number of cars with the average speed of $100 \mathrm{~km} / \mathrm{hr}$ exits from junction 3 is 3172 whereas that of cars with the average speed of $125 \mathrm{~km} / \mathrm{hr}$ is 3585 . It is worth to mention that the total car exit number for the speed limit with $125 \mathrm{~km} / \mathrm{hr}$ is larger than that of speed limit with $100 \mathrm{~km} / \mathrm{hr}$. Therefore, we further analyze the current car number and average speed based on installing a traffic light control and with a speed limit of 125 $\mathrm{km} / \mathrm{hr}$ in each lane. In Table 2 , the red light duration is classified into 4 cases: 0 , 5,10 , and 15 seconds. Table 2 shows that the longer red light duration is the 
fewer current cars have in each lane. Meanwhile, the longer red light duration the more total cars exiting from the end junction. When the red light duration is 0 second, there are 3585 cars to drive off the end junction. When the red light duration is 15 seconds, 4113 cars are counted to drive off the end junction. However, from Table 3, we note that the longer the red light duration the longer waiting time/waiting number. That means all cars are waiting in the entrance ramp so the highway traffic can have good performance. But, for a driver, it is not a good solution because the total time from home to office still takes long. In particular, when the red light duration is 15 seconds, the average waiting time reaches 517.53 seconds and the waiting number of cars is 300 cars as shown in Table 3. Consequently, we suggest that if we set a dynamic speed limit signal in the rush hours to increase speed limit, the performance of the highway will be better.

Table 1: The total exit number of cars and average speed at each lane for different speed limit policies without red light control.

\begin{tabular}{ccccc}
\hline \hline & \multicolumn{2}{c}{ Speed limit $100(\mathrm{~km} / \mathrm{hr})$} & \multicolumn{2}{c}{ Speed limit 125 $(\mathrm{km} / \mathrm{hr})$} \\
\cline { 2 - 5 } Lane no & $\begin{array}{c}\text { Current car } \\
\text { number }\end{array}$ & $\begin{array}{c}\text { Average speed } \\
(\mathrm{km} / \mathrm{hr})\end{array}$ & $\begin{array}{c}\text { Current car } \\
\text { number }\end{array}$ & $\begin{array}{c}\text { Average speed } \\
(\mathrm{km} / \mathrm{hr})\end{array}$ \\
\hline Lane 1 & 1337 & 40.7 & 1341 & 40.6 \\
Lane 2 & 1425 & 37.6 & 1216 & 45.8 \\
Lane 3 & 1304 & 42.0 & 1096 & 51.9 \\
\hline $\begin{array}{c}\text { Total car exit } \\
\text { number in the } \\
\text { end junction }\end{array}$ & & 3172 & & 3585 \\
\hline \hline
\end{tabular}

Table 2: $\quad$ The total exit number of cars and average speed at each lane for different red light durations with speed limit $125(\mathrm{~km} / \mathrm{hr})$.

\begin{tabular}{|c|c|c|c|c|c|c|c|c|}
\hline \multirow[b]{3}{*}{ Lane no } & \multicolumn{8}{|c|}{ Red light duration } \\
\hline & \multicolumn{2}{|c|}{$0 \mathrm{sec}$} & \multicolumn{2}{|c|}{$5 \mathrm{sec}$} & \multicolumn{2}{|c|}{$10 \mathrm{sec}$} & \multicolumn{2}{|c|}{$15 \mathrm{sec}$} \\
\hline & $\begin{array}{c}\text { Current } \\
\text { car } \\
\text { number }\end{array}$ & $\begin{array}{c}\text { Average } \\
\text { speed } \\
(\mathrm{km} / \mathrm{hr})\end{array}$ & $\begin{array}{c}\text { Current } \\
\text { car } \\
\text { number }\end{array}$ & $\begin{array}{c}\text { Average } \\
\text { speed } \\
(\mathrm{km} / \mathrm{hr})\end{array}$ & $\begin{array}{c}\text { Current } \\
\text { car } \\
\text { number }\end{array}$ & $\begin{array}{c}\text { Average } \\
\text { speed } \\
(\mathrm{km} / \mathrm{hr})\end{array}$ & $\begin{array}{c}\text { Current } \\
\text { car } \\
\text { number }\end{array}$ & $\begin{array}{c}\begin{array}{c}\text { Average } \\
\text { speed } \\
(\mathrm{km} / \mathrm{hr})\end{array} \\
\end{array}$ \\
\hline Lane 1 & 1341 & 40.6 & 1267 & 43.5 & 1080 & 52.8 & 867 & 68.2 \\
\hline Lane 2 & 1216 & 45.8 & 1253 & 44.1 & 1071 & 53.3 & 876 & 67.4 \\
\hline Lane 3 & 1096 & 51.9 & 1129 & 50.1 & 969 & 60.0 & 789 & 76.0 \\
\hline $\begin{array}{c}\text { Total exit } \\
\text { car } \\
\text { number }\end{array}$ & \multicolumn{2}{|c|}{3585} & \multicolumn{2}{|c|}{3589} & \multicolumn{2}{|c|}{3825} & \multicolumn{2}{|c|}{4113} \\
\hline
\end{tabular}


Urban Transport XIII: Urban Transport and the Environment in the 21st Century 307

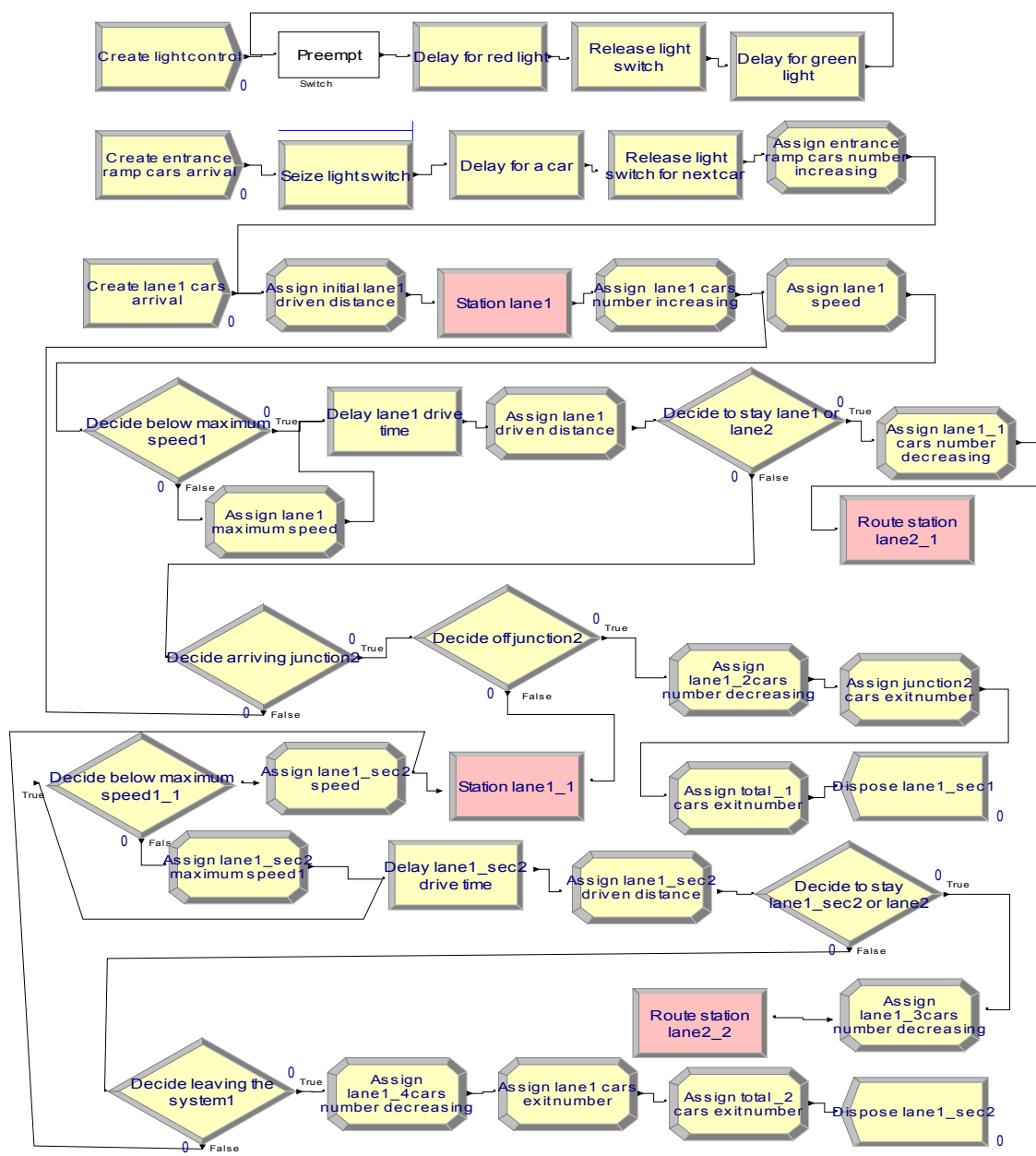

Figure 2: The flow chart of dynamic highway traffic control simulation model. 
308 Urban Transport XIII: Urban Transport and the Environment in the 21st Century

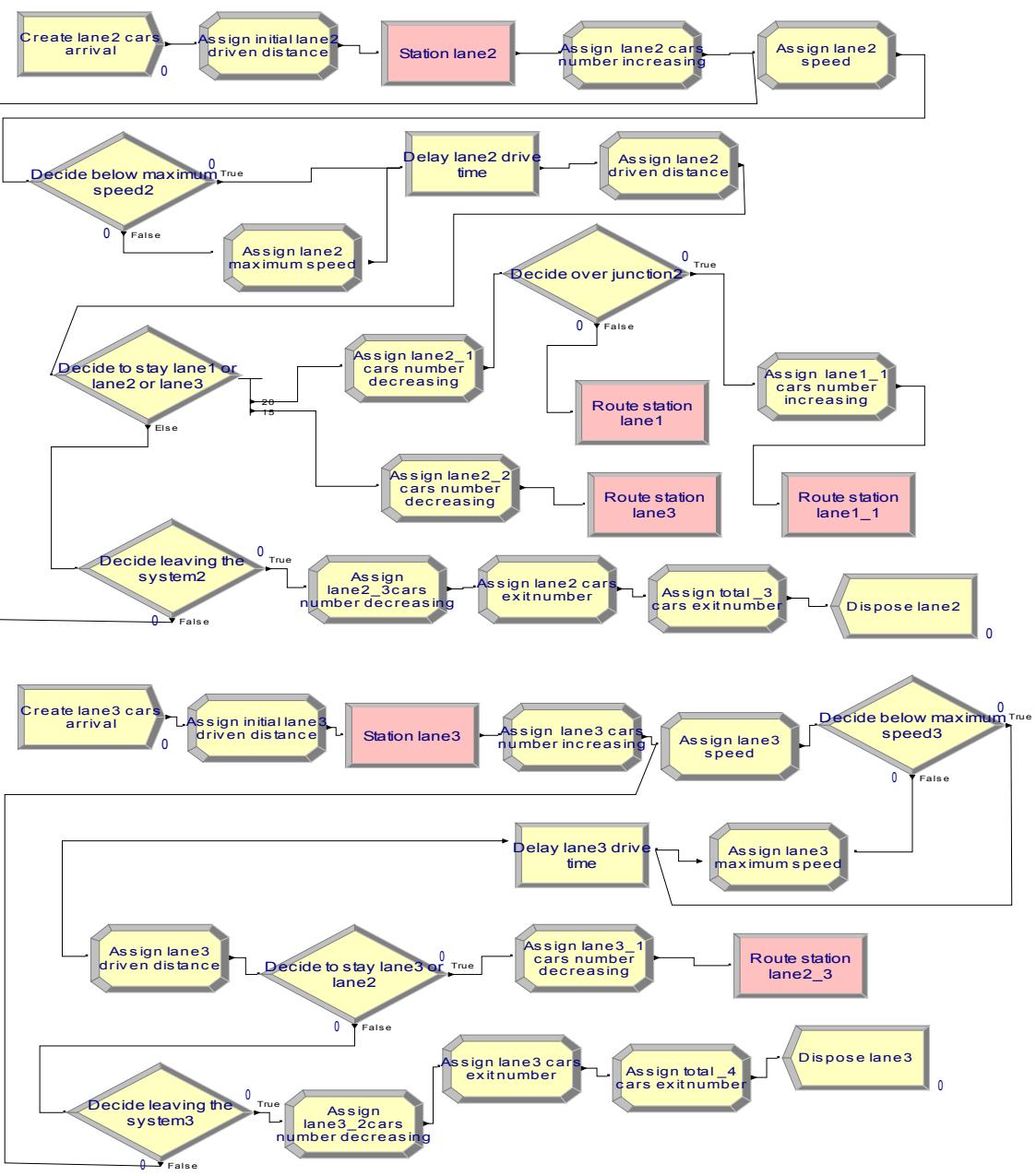

Figure 2: Continued.

Table 3: The average waiting time and waiting number at entrance-ramp for different red light durations with speed limit $125(\mathrm{~km} / \mathrm{hr})$.

\begin{tabular}{ccccc}
\hline \hline & \multicolumn{4}{c}{ Red light duration } \\
\cline { 2 - 5 } Classification & 0 & 5 & $10 \mathrm{sec}$ & $15 \mathrm{sec}$ \\
\hline Average waiting time (sec) & 0 & 1.92 & 257.02 & 517.53 \\
Average waiting number (car) & 0 & 1.12 & 149.03 & 299.65 \\
Total entrance-ramp in lane 1 & 2093 & 2093 & 1800 & 1500 \\
Total waiting time (hr) & 0 & 1.1 & 128.5 & 215.6 \\
\hline \hline
\end{tabular}




\section{Conclusions and future work}

This paper proposes a dynamic highway flow control simulation model for improving traffic congestion problem. To compare performance, we design various cases with different speed limits and different red light durations for improving traffic problem in a highway during rush hours. The simulation results physically prove the efficiency of the simulation model, because the average waiting time and number of cars are dropped down sharply when the red light duration is 0 second. Meanwhile, further analysis also shows if we increase the speed limit in each lane, the total performance of the simulation model will be better. Although this paper presents and analyzes a dynamic highway flow control simulation model, there are still several aspects where we can further consider letting it be more reality. For example, we can extend the simulation model to add some more exit junctions or distances to know the interrelationship between junctions and speed limit. In addition, because we can collect traffic flow and average car speed by using information technology, an expert system of dynamically controlling speed limit for solving highway traffic congestion will be also a major research issue in the future.

\section{References}

[1] Alvarez, L., Hoberto, H., and Toy, C.V., Multi-destination Flow Control in Automated Highway Systems, Transportation Research Part C, pp. 128, 2003.

[2] Chen, Y.L. and Yang, H.H. Minimization of Travel Time and Weighted Number of Stops in a Traffic-light Network, Transportation Research B, 34, 2000, pp. 241-253.

[3] Chen, Y.L. and Yang, H.H. Minimization of Travel Time and Weighted Number of Stops in a Traffic-light Network, European Journal of Operational Research, 144, 2003, pp. 565-580.

[4] Golob, T.F. and Recker, W.W., A Method for Relating Type of Crash to Traffic Flow Characteristics on Urban Freeways, Transportation Research Part A, 38, pp. 53-80, 2004.

[5] Grau, R. and Barcelo, J., An Experience in Demand-responsive Traffic Control, Proceeding of ist Meeting of the Euro Working Group in the Urban Traffic and Transportation, Landshut, Techniczl University of Munich, Germany, 1992.

[6] Hall, R. and Chin, C., Vehicle Sorting for Platoon Formation: Impacts on Highway Entry and Throughput, Transportation Research Part A, 13, pp. 405-420, 2005.

[7] Lee, H.Y., Lee, H.W., and Kim, D., Traffic State of a Model Highway with on-ramp, Physic A, pp. 78-86, 2000.

[8] Mahmoud, M. E. and El-Araby, K., A Robust Dynamic Highway Traffic Simulation Model, Computers and Industrial Engineering, pp. 189-193, 1999. 
[9] Messmer, A., and Papageorgiou, M., Automatic Control Methods Applied to Freeway Network Traffic, 12 IFAC World Congress, Australia, Vol. 9, pp. 233-238.

[10] Qin, X., Ivan, J.N., and Ravishanker, N., Selecting Exposures in Crash Rate Prediction for Two-lane Highway Segments, Accident Analysis \& Prevention, 36, pp. 183-191, 2004.

[11] Schadschneider, A., Cellular Automata Models of Highway Traffic, Physic A, 372, pp. 142-150, 2006.

[12] Schaefer, L., Upchurch, J. and Ashur, S. A., An Evaluation of Freeway Lane Control Signing Using Computer Simulation, Math. Computer Modelling, Vol. 27, No. 9-11, pp. 177-187, 1998.

[13] Stoilova, K. and Stoilov T. Traffic Noise and Traffic Light Control, Transportation Research-D, Vol. 3, No. 6, pp. 399-417, 1998.

[14] US Department of transportation, Congestion Mitigation, http://www.fhwa.dot.gov/congestion/congest2.htm.

[15] Zhang, L., Operational Models and Traffic Control in Intelligent Vehicle/Highway Systems, Ph.D., Princeton University, 1996. 